\title{
The contribution of respiratory pathogens to fatal and non-fatal respiratory hospitalizations: a pilot study of Taqman Array Cards (TAC) in Kenya
}

Henry N. Njuguna ${ }^{1 *}$ D, Sandra S. Chaves ${ }^{1}$, Gideon O. Emukule, Bryan Nyawanda², Victor Omballa², Bonventure Juma', Clayton O. Onyango ${ }^{1}$, Joshua A. Mott ${ }^{1}$ and Barry Fields ${ }^{1}$

\begin{abstract}
Background: Respiratory diseases cause substantial morbidity and mortality worldwide, with sub-Saharan Africa bearing the greatest burden. Identifying etiologies of respiratory disease is important to inform cost effective treatment, prevention and control strategies. Testing for all of the different pathogens that are potentially associated with respiratory illnesses is challenging. We piloted the use of a multi-pathogen respiratory Taqman Array Cards (TAC) to identify pathogens in respiratory samples collected from non-fatal and fatal cases and their matched asymptomatic controls.
\end{abstract}

Methods: This is a case control study comparing viral and bacterial pathogens detected among non-fatal and fatal cases to those detected among age and time matched asymptomatic controls. We used McNemar's test to compare proportions of pathogens detected among cases (non-fatal and fatal) to their matched asymptomatic controls. We used Mann-Whitney test to compare the distribution of median Cycle threshold (Ct) values among non-fatal and fatal cases to their corresponding asymptomatic controls.

Results: There were 72 fatal and 72 non-fatal cases matched to 72 controls. We identified at least one pathogen in 109/144 (76\%) cases and 59/72 (82\%) controls. For most pathogens, the median Ct values were lower among cases (fatal and non-fatal) compared to asymptomatic controls.

Conclusions: Similar rates of pathogen detection among cases and controls make interpretation of results challenging. Ct-values might be helpful in interpreting clinical relevance of detected pathogens using multipathogen diagnostic tools.

Keywords: Respiratory pathogens, Respiratory illness, Multi-pathogen Taqman array cards

\section{Background}

Respiratory illnesses associated with bacteria, fungi or viral infections cause substantial morbidity worldwide. Specific etiology cannot be distinguished on basis of clinical presentation alone. Understanding the relative contribution of different pathogens to severe respiratory illnesses could be an important tool to inform and prioritize specific clinical interventions or public health policies. Nonetheless, testing for all of the different

\footnotetext{
* Correspondence: vkc7@cdc.gov

${ }^{1}$ Centers for Disease Control and Prevention, Nairobi, Kenya

Full list of author information is available at the end of the article
}

pathogens that are potentially associated with respiratory illnesses is challenging.

Molecular-based multi-pathogen diagnostic tests such as Taqman Array cards (TAC) may offer some resolution to the diagnostic challenges of respiratory illnesses, especially in the setting of outbreaks of unknown etiology. These tests are capable of detecting a broad range of pathogens by multiplexing polymerase chain reaction (PCR), and have a short turn-around-time of approximately $3 \mathrm{~h}[1]$. We piloted the use of respiratory TAC to identify pathogens in nasopharyngeal (NP) and oropharyngeal (OP) specimens collected from hospitalized 
patients with respiratory illness (fatal and non-fatal cases) and corresponding matched asymptomatic controls. The hypothesis was that as we moved through the spectrum of disease severity (from asymptomatic to fatal cases), we would see changes in the types and frequency of pathogens detected that could assist with interpretation of disease etiology.

\section{Methods}

\section{Participants and definitions}

Clinical data on patients' specimens collected from 1st Aug 2009 through 31st Jul 2011 at Siaya County Referral Hospital (Siaya CRH) and Lwak Mission Hospital (Lwak $\mathrm{MH}$ ) were obtained. Both hospitals are located in rural western Kenya, and surveillance activities are operated by the Kenya Medical Research Institute (KEMRI) in collaboration with the U.S. Centers for Disease Control and Prevention in Kenya (CDC-Kenya) [2, 3]. At Siaya $\mathrm{CRH}$, for consenting patients hospitalized with acute respiratory illness (cases) demographic data were collected using structured questionnaires if at least one of the following symptoms had been present during the previous 14 days: cough, difficulty breathing, sore throat, or chest pain. In addition, NP/OP specimens were collected for TAC testing. Cases were further classified as non-fatal if they were discharged from hospital alive or fatal if they died during hospitalization. We also enrolled asymptomatic controls among those visiting the outpatient clinic at Lwak MH due to non-infectious conditions. Reasons for outpatient visit could include drug refills, vaccination services or well-visits; persons accompanying sick relatives were also approached for enrollment as control. Controls were excluded if they reported respiratory symptoms, fever or diarrhea within 14 days prior to their clinic visit [4]. Lwak $\mathrm{MH}$ is located at approximately $30 \mathrm{~km}$ from Siaya CRH, serving a similar population [5]. Initially, we identified non-fatal cases and matched them individually with fatal cases based on age and date of admission (within +/- 30 days) at a ratio of one-to-one. Then we identified a similar number of asymptomatic controls within the same time-frame as the cases and matched them in similar fashion (based on age and date of admission) to the previously matched non-fatal and fatal cases. The age-matching was done considering up to 4 months difference for those aged $<18$ years, and a 5 year-band difference for those $\geq 18$ years.

\section{Laboratory methods}

$\mathrm{NP} / \mathrm{OP}$ swabs were combined in a single cryovial containing $1 \mathrm{ml}$ viral transport media (VTM). These specimens were transported on the same day in cool boxes at $2-8{ }^{\circ} \mathrm{C}$ to the KEMRI laboratory (Kisumu, Kenya) where they were stored at $-80{ }^{\circ} \mathrm{C}$ until processed. Fifty microliters of NP/OP specimen was tested using respiratory
TAC for viral targets [influenza virus type A, influenza virus type $B$, respiratory syncytial virus (RSV), parainfluenza virus type I (PIV-1), PIV-2, PIV-3, Human metapneumovirus (HMPV), Rhinovirus /Enterovirus (RV/EV), and Adenovirus (AV)] and bacterial targets [Streptococcus pneumoniae, Haemophilus influenzae pan, Mycoplasma pneumoniae and Bordetella pertussis] [6] [for more details, see Additional file 1: Fig. S1 (Schematic diagram of the two versions of Taqman array cards (TAC) used in the study) in supplementary material]. A positive result in TAC for each pathogen was defined as cycle threshold $(\mathrm{Ct})$ values $<40$.

\section{Statistical analysis}

We stratified the study population by age group and calculated the prevalence of each virus detected from NP/ OP specimens collected from non-fatal and fatal cases and asymptomatic controls. We used McNemar's test to compare the proportions of pathogens detected among cases (non-fatal and fatal) to their matched asymptomatic controls. We used odds ratios (OR) to determine pathogens associated with cases (non-fatal and fatal) compared to controls. We used the median $\mathrm{Ct}$ value as a proxy of pathogen density in the NP/OP specimen [7], using the Mann-Whitney test to compare the distribution of the median $\mathrm{Ct}$ values between non-fatal and fatal cases and their corresponding asymptomatic controls separately for each pathogen identified. All statistical analysis was carried out using Stata version 13.0 (StataCorp. 2013. Stata Statistical Software: Release 13. College Station, TX: StataCorp LP) and $p$-values $<0.05$ were considered to statistically significant.

\section{Results}

There were equal number of fatal and non-fatal cases and matched controls $(n=72$ each). Of the total studypatients $(n=216), 102(47 \%)$ were aged $<5$ years and 114 (53\%) were $\geq 5$ years. For each age category, the median age was 1.5 years (range 3 - 59 months) for those $<5$ years and 36 years (range 5 - 90 years) for those $\geq 5$ years. Among non-fatal cases, 61 (85\%) presented with cough, $40(56 \%)$ with difficulty in breathing, 17 (24\%) with chest pain and $10(29 \%)$ with sore throat (these symptoms were not mutually exclusive). Among fatal cases, 63 (88\%) presented with cough, 45 (63\%) with difficulty in breathing, $19(26 \%)$ with chest pain, and 13 (41\%) with sore throat.

Using TAC, we detected at least one pathogen from $109 / 144$ (77\%) cases. There was no difference in pathogen distribution among non-fatal and fatal cases based on the presenting symptoms. Overall, among all cases, there was a higher percent of viral co-detection $(11 \%$ had 2 viruses and $6 \%$ had $\geq 3$ viruses detected) compared to bacteria co-detection (7\% had 2 bacteria detected and 
$1 \%$ had $\geq 3$ bacteria detected). There were more viral codetections among non-fatal cases, with $6(8 \%)$ having $\geq 3$ viruses detected compared to $3(4 \%)$ among fatal cases; but total number of detected pathogens were similar in the two groups ( $n=54$ vs $n=55$ among non-fatal and fatal cases respectively). Children $<5$ years had a higher percent of pathogens detected than those $\geq 5$ years: $100 \%$ vs $52 \%$ among non-fatal, and $88 \%$ vs $66 \%$ among fatal cases, respectively (Additional file 1: Table S1). We detected at least one pathogen from 59/72 (82\%) asymptomatic controls with higher co-detection of bacteria ( $44 \%$ had 2 bacteria) compared to viruses (15\% had 2 viruses). Only 4/72 (6\%) asymptomatic controls developed an illness after sample collection. One developed respiratory symptoms 6 days after being swabbed (originally had rhinovirus detected in the study sample); one developed fever 3 days after being swabbed (had no pathogens detected); one developed unspecified non-respiratory symptoms 3 days after sample collection (had enterovirus, Haemophilus influenzae and S. pneumoniae detected in the study sample); and one developed respiratory symptoms 1 day after the NP/OP sample being collection (had no pathogens detected).

Enterovirus/rhinoviruses were the most commonly detected viruses: $40 \%$ among non-fatal cases, $46 \%$ among fatal cases and 39\% among asymptomatic controls (Additional file 1: Table S1). There was no difference in enterovirus/rhinovirus detection between non-fatal and asymptomatic controls $(p=0.87)$ and fatal and asymptomatic controls $(p=0.40)$. The most commonly detected bacteria was S. pneumoniae: $57 \%$ among non-fatal cases, $61 \%$ among fatal cases and 58\% among asymptomatic controls. There was no difference in S.pneumoniae detection between non-fatal and asymptomatic controls $(p=0.09)$ and fatal and asymptomatic controls $(p=0.08)$ (Fig. 1). H. influenzae were less likely to be detected among non-fatal cases (OR 0.08, 95\% CI 0.020.25 ) and fatal cases (OR $0.05,95 \%$ CI $0.12-0.22$ ) compared to their respective asymptomatic controls (Additional file 1: Table S2). However, for almost all more frequently detected pathogens (i.e., adenovirus, PIV-3, RSV, enterovirus/rhinovirus, Streptococcus pneumoniae, and Haemophilus influenzae), median Ct values were lower for cases (fatal and non-fatal) than controls, though these differences were not statistically significant (Fig. 2). Enterovirus/rhinovirus had significantly lower median $\mathrm{Ct}$ values among fatal cases compared to asymptomatic controls $(p<0.01)$; and $S$. pneumoniae, had significantly lower $\mathrm{Ct}$ values among non-fatal cases compare to asymptomatic controls $(p<0.01)$.

\section{Discussion}

We detected multiple pathogens in NP/OP specimens collected among non-fatal and fatal cases and asymptomatic

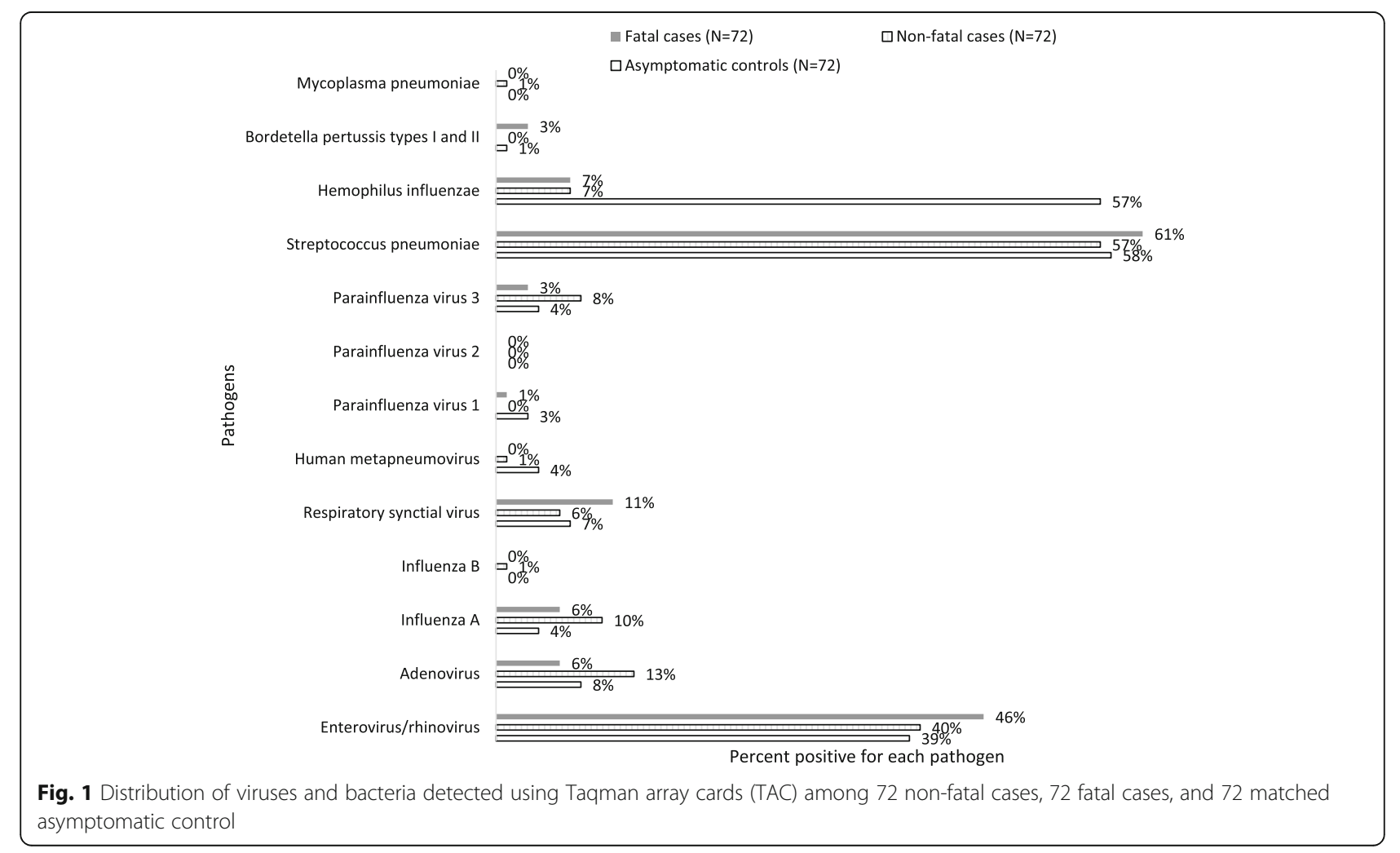




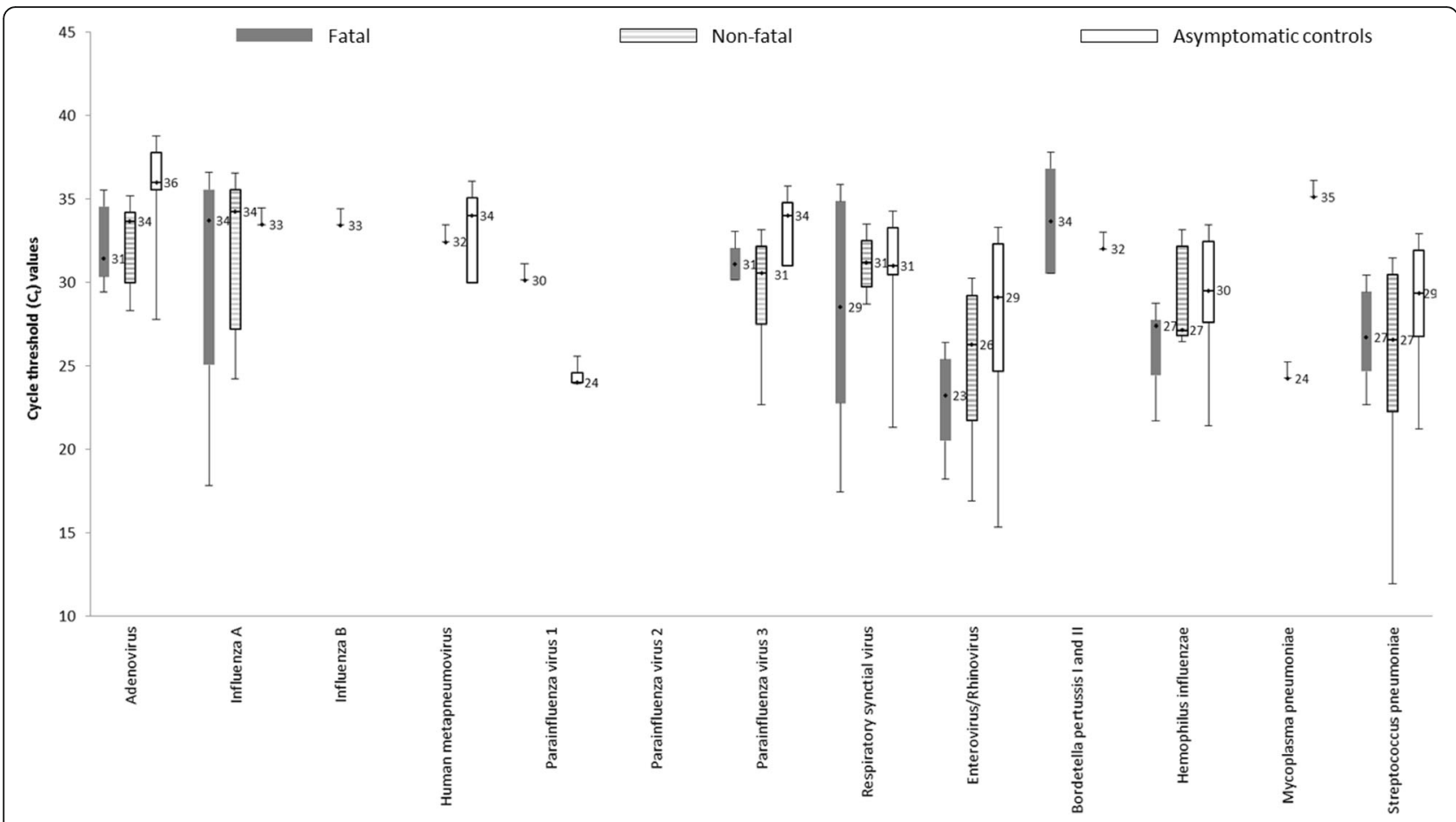

Fig. 2 Box plot showing cycle threshold (Ct) values for agents targeted by Taqman Array Card (TAC) among 72 non-fatal cases, 72 fatal cases and 72 asymptomatic controls. Boxes show the median (dot and number) and the 25th and 75th percentiles and bars show the 10th and 90th percentiles. *Pathogens whose median Ct value is significantly lower among non-fatal or fatal cases compared to asymptomatic controls using Mann-Whitney test (Enterovirus/Rhinovirus median Ct: fatal-cases (23.23) vs asymptomatic controls (29.10), $p$ value $<0.01$; S.pneumoniae median Ct: non-fatal cases (26.60) vs asymptomatic controls (29.34), $p$ value $<0.01$ )

controls. Careful interpretation of results is needed since over $80 \%$ of participants enrolled as asymptomatic controls had at least one pathogen identified. Ct values may play an important role when assessing etiological role of pathogens detected in similar rates among cases and controls.

Enterovirus/rhinovirus, RSV and adenovirus were the leading viral pathogens detected from non-fatal and fatal cases, similar to previous reports $[4,8,9]$. S. pneumoniae was the leading bacterial pathogen detected in both nonfatal and fatal cases $(60 \%)$. These rates were within the range observed from other studies, i.e., $55-75 \%[8,10]$. We did not find substantial differences in pathogen detection in cases (fatal and non-fatal) compared to controls. Other studies have shown pathogens such as influenza virus, RSV, rhinovirus, HMPV and Streptococcus pneumoniae being detected more frequently in cases compared to asymptomatic controls $[9,11,12]$. In fact, in our study, $H$. influenzae had a 5 fold increase in detection rates among asymptomatic controls compared to cases - even considering bacteria carriage as common in the population, this may need further investigation. The 14 day symptom-free period required for controls could have been too short, some patients may be convalescent, coming for drug refills. On the other hand, cases could have the distribution of bacterial pathogens in the respiratory tract altered by previous antibiotic treatment [13].

Despite the lack of statistical significance, the median $\mathrm{Ct}$ values for adenovirus, PIV-3, RSV, enterovirus/rhinovirus, Streptococcus pneumonia, and Haemophilus influenzae were lower among cases (fatal and non-fatal) compared to asymptomatic controls; this could suggest an etiologic contribution to the clinical presentation. Previous studies have demonstrated that $\mathrm{Ct}$ values could be a proxy for assessing concentration of pathogens and lower values have been associated with disease severity [7]. Both fatal and non-fatal cases positive for enterovirus/rhinovirus and S. pneumoniae had significantly lower median Ct values than asymptomatic controls. Assuming that $\mathrm{Ct}$ values could be a proxy for pathogen density, enterovirus/rhinovirus and S. pneumoniae could have played a role in the pathogenesis of respiratory illness among case-patients. High density of S. pneumoniae in the nasopharynx, for instance, has been associated with pneumonia [14]. For future studies using multi-pathogen diagnostic platform, the strength of association between pathogen detected with respiratory illness may have to be assessed relative to pathogen loads in order to infer etiology. 
There were a number of limitations to this study. NP/ OP swabs are non-sterile-site specimens. Using a highly sensitive molecular technique on non-sterile specimens can detect small amounts of nucleic material even in asymptomatic individuals who are either incubating or recovering from infection [15]. Our case definition was quite broad. It is possible that some patients presented with other illnesses such as malaria (endemic in this area) or with nonspecific respiratory symptoms were misclassified as having a respiratory disease. This may have reduced the chances of detecting respiratory disease pathogens among the cases. The low sample size in our study does not afford the statistical power needed to determine an association between most pathogens detected and respiratory disease, despite the trend toward some pathogens of lower $\mathrm{Ct}$ values (i.e., high concentration) amongst non-fatal and fatal cases compared to controls.

\section{Conclusions}

Multi-pathogen molecular diagnostics such as TAC can be useful for investigation of respiratory outbreaks of unknown origin and to assess the distribution of pathogens in a defined population. However, the attribution of pathogens as a cause of respiratory disease using this technology needs to be further evaluated. This study supports previous published studies suggesting the use of $\mathrm{Ct}$ values to define clinically relevant pathogen concentrations. Further studies are warranted to investigate and establish appropriate pathogen-specific Ct cutoff values indicative of disease.

\section{Additional file}

Additional file 1: Figure S1. Schematic diagram of the two versions of Taqman array cards (TAC) used in the study. Table S1. Viral and bacterial pathogens detected using Taqman array cards (TAC) among non-fatal and fatal cases and asymptomatic controls, by age group, western Kenya, 2009-11. Table S2. Distribution of respiratory pathogens among cases (non-fatal and fatal) and corresponding asymptomatic controls in rural western Kenya, 2009-11. (DOCX 614 kb)

\section{Abbreviations}

AV: Adenovirus; CDC: Centers for Disease Control and Prevention; CRH: County Referral Hospital; Ct: Cycle threshold; EV: Enterovirus; HMPV: Human metapneumovirus; KEMRI: Kenya Medical Research Institute; MH: Mission Hospital; NP: Nasopharyngeal; OP: Oropharyngeal; OR: Odds ratio; PCR: Polymerase chain reaction; PIV: Parainfluenza virus; RSV: Respiratory syncytial virus; RV: Rhinovirus; TAC: Taqman Array Cards; VTM: Viral transport media

\section{Acknowledgments}

We would like to thank the surveillance officers at Siaya County Referral Hospital and Lwak Mission Hospital for their great work in identifying cases and control, collecting data and clinical specimens. We appreciate the KEMRI-CDC data staff for cleaning data and the KEMRI-CDC laboratory staff for testing specimens collected.

\section{Funding}

This work was supported by the Centers for Disease Control and Prevention (CDC).

\section{Availability of data and materials}

The authors confirm that, in accordance with Institutional Review Board policies that protect confidential data, some access restrictions apply to the data underlying the findings. Ethical restrictions prevent public deposition of data. Data are available upon written request to the KEMRI, SERU Committee for researchers who meet the criteria for access to confidential data. The request should include specific aims, hypotheses to be addressed, specific variables that are directly relevant to the proposed analysis, and an explanatory statement on how requested data sets will answer the proposed question(s). Requests should be sent to KEMRI-SERU, P.O Boxes 54,840-00200 Nairobi Kenya, and Email: director@KEMRl.org.

\section{Authors' contributions}

HN: Participated in developing the study design, data analysis, drafted and finalized the manuscript. SC: Participated in data analysis and interpretation and manuscript review. GE and BN: Participated in study design, data analysis and interpretation and manuscript review. VO, BJ and CO: Contributed on laboratory testing, interpretation of TAC results and manuscript writing. JM and BF: Participated in study conception, study design and manuscript review. All authors read and approved the final manuscript.

\section{Ethics approval and consent to participate}

The study protocol and consent forms were reviewed and approved by the KEMRI scientific ethics review unit (SSC-1801) and (SSC-932) and CDC's institutional review board (CDC-3308) and (CDC-4566). A written informed consent was obtained from all participants (or guardians of those $<18$ years), and written assent was obtained for patients 13-17 years.

\section{Consent for publication}

Not applicable.

\section{Competing interests}

The authors declare that they have no competing of interest.

\section{Publisher's Note}

Springer Nature remains neutral with regard to jurisdictional claims in published maps and institutional affiliations.

\section{Author details}

${ }^{1}$ Centers for Disease Control and Prevention, Nairobi, Kenya. ${ }^{2}$ Kenya Medical Research Institute, Nairobi, Kenya.

Received: 4 May 2017 Accepted: 17 August 2017

Published online: 25 August 2017

\section{References}

1. Kodani M, Yang G, Conklin LM, Travis TC, Whitney CG, Anderson LJ, Schrag SJ, Taylor TH Jr, Beall BW, Breiman RF, et al. Application of TaqMan lowdensity arrays for simultaneous detection of multiple respiratory pathogens. J Clin Microbiol. 2011;49:2175-82.

2. Emukule GO, Khagayi S, McMorrow ML, Ochola R, Otieno N, Widdowson MA, Ochieng M, Feikin DR, Katz MA, Mott JA. The burden of influenza and RSV among inpatients and outpatients in rural western Kenya, 2009-2012. PLoS One. 2014:9:e105543.

3. Feikin DR, Olack B, Bigogo GM, Audi A, Cosmas L, Aura B, Burke H, Njenga MK, Williamson J, Breiman RF. The burden of common infectious disease syndromes at the clinic and household level from population-based surveillance in rural and urban Kenya. PLoS One. 2011:6:e16085.

4. Feikin DR, Njenga MK, Bigogo G, Aura B, Aol G, Audi A, Jagero G, Muluare PO, Gikunju S, Nderitu L, et al. Etiology and incidence of viral and bacterial acute respiratory illness among older children and adults in rural western Kenya, 2007-2010. PLoS One. 2012;7:e43656.

5. Odhiambo FO, Laserson KF, Sewe M, Hamel MJ, Feikin DR, Adazu K, Ogwang S, Obor D, Amek N, Bayoh N, et al. Profile: the KEMRI/CDC health and demographic surveillance system-western Kenya. Int J Epidemiol. 2012; 41:977-87.

6. Diaz MH, Waller JL, Napoliello RA, Islam MS, Wolff BJ, Burken DJ, Holden RL, Srinivasan V, Arvay M, McGee L, et al. Optimization of multiple pathogen detection using the TaqMan Array card: application for a population-based study of neonatal infection. PLoS One. 2013;8:e66183. 
7. Houben ML, Coenjaerts FE, Rossen JW, Belderbos ME, Hofland RW, Kimpen $J$, Bont L. Disease severity and viral load are correlated in infants with primary respiratory syncytial virus infection in the community. J Med Virol. 2010:82:1266-71.

8. Simusika P, Bateman AC, Theo A, Kwenda G, Mfula C, Chentulo E, Monze M. Identification of viral and bacterial pathogens from hospitalized children with severe acute respiratory illness in Lusaka, Zambia, 2011-2012: a crosssectional study. BMC Infect Dis. 2015;15:52.

9. Jain S, Williams DJ, Arnold SR, Ampofo K, Bramley AM, Reed C, Stockmann C, Anderson EJ, Grijalva CG, Self WH, et al. Community-acquired pneumonia requiring hospitalization among U.S. children. N Engl J Med. 2015;372:835-45.

10. Verhagen LM, Gomez-Castellano K, Snelders E, Rivera-Olivero I, Pocaterra L, Melchers WJ, de Waard JH, Hermans PW. Respiratory infections in Enepa Amerindians are related to malnutrition and Streptococcus Pneumoniae carriage. J Inf Secur. 2013;67:273-81.

11. Breiman RF, Cosmas L, Njenga M, Williamson J, Mott JA, Katz MA, Erdman DD, Schneider E, Oberste M, Neatherlin JC, et al. Severe acute respiratory infection in children in a densely populated urban slum in Kenya, 20072011. BMC Infect Dis. 2015;15:95.

12. Benet T, Sylla M, Messaoudi M, Sanchez Picot V, Telles JN, Diakite AA, Komurian-Pradel F, Endtz H, Diallo S, Paranhos-Baccala G, Vanhems P. Etiology and factors associated with pneumonia in children under 5 years of age in Mali: a prospective case-control study. PLoS One. 2015;10:e0145447.

13. Pettigrew MM, Laufer AS, Gent JF, Kong Y, Fennie KP, Metlay JP. Upper respiratory tract microbial communities, acute otitis media pathogens, and antibiotic use in healthy and sick children. Appl Environ Microbiol. 2012;78: 6262-70.

14. Vu HT, Yoshida LM, Suzuki M, Nguyen HA, Nguyen CD, Nguyen AT, Oishi K, Yamamoto T, Watanabe K, Vu TD. Association between nasopharyngeal load of Streptococcus Pneumoniae, viral coinfection, and radiologically confirmed pneumonia in Vietnamese children. Pediatr Infect Dis J. 2011;30:11-8.

15. Jansen RR, Wieringa J, Koekkoek SM, Visser CE, Pajkrt D, Molenkamp R, de Jong MD, Schinkel J. Frequent detection of respiratory viruses without symptoms: toward defining clinically relevant cutoff values. J Clin Microbiol. 2011;49:2631-6.

\section{Submit your next manuscript to BioMed Central and we will help you at every step:}

- We accept pre-submission inquiries

- Our selector tool helps you to find the most relevant journal

- We provide round the clock customer support

- Convenient online submission

- Thorough peer review

- Inclusion in PubMed and all major indexing services

- Maximum visibility for your research

Submit your manuscript at www.biomedcentral.com/submit 